\title{
The dopaminergic system of ventral hippocampus is involved in the
} anxiety related behavior Sahand Babapoor-Farrokhran*1, Mohammad-Reza Zarrindast ${ }^{1,3,4}$ and Ameneh Rezayof ${ }^{2}$

Address: ${ }^{1}$ Department of Pharmacology, School of Medicine and Iranian Center for Addiction Studies, Tehran University of Medical Sciences, Tehran, Iran, ${ }^{2}$ School of Biology, University College of Science, University of Tehran, Tehran, Iran, ${ }^{3}$ Institute for Studies in Theoretical Physics and Mathematics, School of Cognitive Sciences, Tehran, Iran and ${ }^{4}$ Institute for Cognitive Science Studies, Tehran, Iran

* Corresponding author

from International Society on Brain and Behaviour: 3rd International Congress on Brain and Behaviour Thessaloniki, Greece. 28 November - 2 December 2007

Published: 17 April 2008

Annals of General Psychiatry 2008, 7(SuppI I):S232 doi:10.1 186/I744-859X-7-SI-S232

This abstract is available from: http://www.annals-general-psychiatry.com/content/7/SI/S232

(c) 2008 Babapoor-Farrokhran et al.; licensee BioMed Central Ltd.

\section{Background}

In the previous studies, it has been shown that dopaminergic mechanisms are related to the production and elaboration of acute and chronic stress. The ventral hippocampus is one of the important brain sites involved in modulation of fear and anxiety.

\section{Materials and methods}

To test the effects of dopaminergic system in the ventral hippocampus $(\mathrm{VH})$, we investigated the effect of apomorphine, sulpiride (D2 receptor antagonist) and SCH 23390 (D1 receptor antagonist) microinjections into the $\mathrm{VH}$ on the behaviors displayed by male Wistar rats in the elevated plus-maze. In these experiments, animals weighting $220 ? 280 \mathrm{~g}$ at the time of surgery were used. Eight animals were used in each group of experiments. After bilaterally cannulating of the animals in the VH by stereotaxic instrument, they were allowed to recover 1-week before behavioral testing. All procedures were carried out in accordance with institutional guidelines for animal care and use. Test videotapes were scored for conventional indices of anxiety (percent open arm entries / time) and locomotor activity (closed arm entries).

\section{Results}

Bilateral intra- $\mathrm{VH}$ injections of the different doses of apomorphine $(0.1,0.5,1$ microgram per rat) increased the percentage of open arm time (\%OAT) and open arm entries $(\% \mathrm{OAE})$. The results suggest that apomorphine produces a significant anxiolytic effect without any changes in the locomotor activity. Similarly, intra-VH microinjections of sulpiride $(0.2,1,5$ micrograms per rat), but not SCH 23390 (0.01, 0.1, 1 microgram per rat), has increased (\%OAE) indicating an anxiolytic action. The observed effect of sulpiride may be mediated through dopamine release from the dopaminergic terminals in the $\mathrm{VH}$.

\section{Conclusions}

In conclusion, dopaminergic system of the VH can elicit anxiolytic behavior.

\section{Acknowledgements}

The project has been supported by Tehran university of medical sciences. This abstract is being presented on behalf of Dr. Zarrindast and Dr. Rezayof.

\section{References}

I. Garcia AM, Martinez R, Brandão ML, Morato S: Effects of apomorphine on rat behavior in the elevated plus-maze. Physiol Behav 2005, 85(4):440-7.

2. Rostami P, Hajizadeh-Moghaddam A, Zarrindast MR: The effects of histaminergic agents in the ventral hippocampus of rats in the plus-maze test of anxiety-like behaviours. Physiol Behav 2006, 87:89I-896. 